\title{
MODELOS DE PREVENCIÓN EN LA LUCHA CONTRA EL VIH/SIDA
}

\author{
John Harold Estrada M.*
}

Resumen: Este artículo describe tres modelos teóricos utilizados para afrontar la prevención de la transmisión del VIH en estos años. Estos se han desarrollado de manera secuencial, responden a distintas ópticas de la prevención, representa cada uno un mayor nivel de inclusión con relación al anterior y son utilizados en ocasiones de manera ecléctica, sin responder a las necesidades particulares de las poblaciones sujeto de las acciones. Finalmente, el documento analiza algunas lecciones aprendidas.

Palabras clave: VIH, SIDA, modelos de prevención

\section{PREVENTION MODELS IN THE FIGHT AGAINST HIV/AIDS}

This article describes three theoretical models to face HIV prevention of transmission in these years. These have been developed sequentially, answering to different prevention views, each one representing a greater inclusion level with respect to the previous one and they are used occasionally in an eclectic way without responding to the particular subject population needs. Finally, the document analyzes some lessons learned.

Key words: HIV, AIDS, prevention models

\section{MODELOS DE PREVENÇÃO NA LUTA CONTRA O VIH/AIDS}

Resumo: Este artigo descrebe três modelos teóricos utilizados para enfrentar a prevenção da transmissão do HIV nestes anos. Estes se desenvolveram de maneira sequencial, respondendo a distintas óticas de prevenção e representam cada um um nível maior de inclusão com relação ao anterior. São utilizados em ocasiões de maneira eclética, sem responder às necessidades particulares das populações, sujeito das ações. Finalmente, o documento analisa algumas lições aprendidas.

Palavras chave: HIV /AIDS, modelos de prevenção

* Profesor Asociado de la Facultad de Odontología y Escuela de Estudios de Género, Universidad Nacional de Colombia. Colombia Correspondencia: estradamontoya@hotmail.com 


\section{Introducción}

La epidemia de infección por el VIH se ha configurado bajo el paradigma del estigma, la discriminación y la exclusión social. Esto no sólo respondió a posturas ideológicas, sino también al proceso histórico de desarrollo y madurez de la comunidad gay/lésbica en los años 80 , en particular en los países del primer mundo, que consolidó una agenda política centrada en el reconocimiento de sus derechos civiles y humanos fundamentales. La infección por el VIH aparece en Estados Unidos precisamente cuando el movimiento gay alcanza su madurez política, momento aprovechado por el ultraconservador gobierno de Ronald Reagan para implementar estrategias de vigilancia epidemiológica que condujeron a reforzar el discurso moral y jurídico y a la exclusión y discriminación de las personas viviendo con VIH/SIDA. Más adelante surgieron las pruebas diagnósticas obligatorias para el VIH, la persecución y el acoso de la comunidad gay que vio disminuidas sus conquistas políticas y sociales.

Posteriormente, la preeminencia del discurso "El SIDA es problema de todos" trajo aparejados problemas, ya que se dejó de hacer intervenciones focalizadas en grupos específicos $\mathrm{y}$, por esto, muchas personas empezaron a considerar el VIH como problema ajeno o de "otros". Las políticas implementadas podrían ser calificadas de heterocentradas y adultocéntricas, lo cual dejó fuera a numerosos hombres y mujeres de diferentes edades y orientaciones sexuales. A partir de ese momento, la infección del VIH/SIDA ha continuado devastando a los grupos de hombres que tienen sexo con otros hombres (HSH) y la falta y/o la inoperancia de las acciones gubernamentales han permitido que los indicadores epidemiológicos de prevalencia en este subgrupo poblacional se disparen en toda América Latina y el Caribe hasta valores entre el 5 y el $20 \%$ en algunas ciudades de la región.
Por parte del programa de SIDA de las Naciones Unidas (ONUSIDA), se estima que para diciembre de 2003 se reportaron más de 62 millones de personas infectadas por el VIH desde el inicio de la pandemia, de los cuales 5 millones contrajeron la infección durante el último año, entre ellas 800.000 niños y niñas. Un total de 42 millones de personas viven actualmente con el VIH/SIDA y, de no recibir tratamiento adecuado, pasarán a engrosar las filas de defunciones por causa de la enfermedad(1).

En América Latina y el Caribe la epidemia está bien consolidada y asume muchas facetas, dada la diversidad en los patrones demográficos y de desarrollo de los países de la región, por lo cual existe el peligro de que se propague de forma rápida en ausencia de respuestas nacionales eficaces. Se calcula que aquí viven con el VIH cerca de dos millones de personas, donde se incluyen las 200.000 personas que se infectaron en 2004. En Haití, Guyana, Belice y República Dominicana la prevalencia de VIH estimada en mujeres embarazadas es igual o superior al 1\%; en estas zonas la epidemia está muy arraigada en la población general y se propaga principalmente a través del coito heterosexual, mientras que en Colombia, México, Perú y el Cono Sur se producen más infecciones en hombres que tienen sexo con hombres (HSH), categoría que sigue siendo arbitraria epidemiológicamente, pero que sirve para agrupar todas las prácticas sexuales entre varones, tengan o no una identidad sexual de tipo homosexual. Para algunos de estos países, el VIH/ SIDA es la primera causa de mortalidad, y sólo se colocan por detrás de los países del África Subsahariana. En la región se combinan los factores de desarrollo socioeconómico desigual (en términos de necesidades básicas insatisfechas, porcentaje de analfabetismo y concentración de la riqueza y de la tierra en pocas manos), la gran movilidad de la población y la situación de estigma y discriminación que ex- 
perimentan grupos poblacionales (en particular los hombres que tienen sexo con hombres) como situaciones que favorecen la propagación del VIH(2).

A pesar de que, en el planeta, el $75 \%$ de las nuevas infecciones se producen entre hombres heterosexuales, en nuestro continente se presentan simultáneamente aumentos en las tasas de incidencia en hombres homosexuales y otros HSH y heterosexuales, pero dado el menor tamaño poblacional de la comunidad de $\mathrm{HSH}$ frente a la de hombres heterosexuales, las repercusiones en este grupo son de mayor proporción e impacto y requieren acciones inmediatas y contundentes.

La prevalencia de la infección por el VIH en HSH en algunas ciudades de América Latina y Centroamérica oscilan entre el $5 \%$ y el $20 \%$, valores muy por encima del observado en estudios realizados en la población general de las mismas ciudades o países, lo cual permite afirmar que en muchos de nuestros países (y Colombia no es la excepción) la epidemia se encuentra concentrada en poblaciones de hombres que tienen sexo con hombres. Según los reportes de ONUSIDA, América Latina aporta el mayor número de infecciones nuevas de VIH en HSH después de Estados Unidos de América.

El hecho de que se asumiera durante años una postura de cierta naturalización frente a la mayor afección de grupos de HSH, sumado a la situación de estigma y discriminación que experimentan estos hombres en la mayor parte de nuestro países, podría explicar en parte por qué, pese al paso del tiempo y a los esfuerzos de ONG's y otros colectivos que agrupan hombres gay o de diversas orientaciones sexuales, no se ha logrado controlar la infección y pareciera que, por el contrario, está lejos de lograrse.

El concepto de prevención no corresponde a una categoría única, sino que, por el contrario, refleja las diferentes visiones de la realidad social imperantes en un momento determinado y se considera con un carácter provisional en ausencia de una herramienta definitiva para controlar la transmisión del virus. La prevención de la infección se torna insuficiente y requiere de una lógica de anticipación mayor que en cualquier situación conocida anteriormente. Ante una epidemia tan cambiante, con tantos perfiles coexistiendo, tantas situaciones de inequidad y estigmatización, ¿cómo brindar una perspectiva de prevención homogénea y transparente?

Esquemáticamente y con fines didácticos, podemos describir tres modelos teóricos que se han utilizado para afrontar la prevención de la transmisión del VIH en estos años. Éstos se han desarrollado de manera secuencial, responden a distintas ópticas de la prevención, representa cada uno un mayor nivel de inclusión con relación al anterior y son utilizados en ocasiones de manera ecléctica, sin responder a las necesidades particulares de las poblaciones sujeto de las acciones.

\section{Modelo epidemiológico-conductual}

En la década de los años ochenta, la aparición de la epidemia del VIH/SIDA se consideró como un hecho social y de salud pública, que tuvo efectos negativos sobre las representaciones y valoraciones de la sexualidad y el erotismo. El surgimiento de la epidemia determinó un retroceso en relación con las formas de entender la sexualidad y el erotismo, y propició el restablecimiento de las ideas esencialistas y normativas sobre los comportamientos sexuales. Esta situación trajo consigo la intolerancia y la marginación de las minorías sexuales; la estigmatización de las identidades sexuales y prácticas eróticas que difieren del paradigma heterosexual.

La respuesta de los diferentes gobiernos, para contrarrestar los efectos nefastos de la epi- 
demia, se tradujo en el diseño e implementación de políticas públicas por parte de sus sistemas nacionales o locales de salud. El examen de estas políticas gubernamentales iniciales permite comprender por qué las primeras acciones de prevención estuvieron signadas por un enfoque que denominamos epidemiológicoconductual. Debemos reconocer que fueron estas políticas, desde sus miradas normativa y prescriptiva, las principales promotoras de las ideas que relacionaron la transmisión del VIH con las identidades homosexuales. La finalidad de los discursos médicos no era únicamente contrarrestar los factores de riesgo, sino marcar los parámetros que distinguían el ejercicio de una sexualidad normal de una sexualidad anormal.

La asociación SIDA/homosexualidad produjo, como consecuencia inevitable, que los programas de prevención y las acciones educativas se dirigieran, casi en su totalidad, a la población de hombres homosexuales (denominados en su momento "grupos de riesgo"). Esta homologación fue decisiva para configurar un discurso moralista sobre dichas orientaciones y para considerar la propagación de la infección como resultado de comportamientos sexuales desviados. Tal hecho se confirma con la no inclusión de las conductas heterosexuales en la caracterización de los factores de riesgo; es decir, no reconociendo a los hombres heterosexuales como sujetos que de igual manera están expuestos al contagio y pueden ser transmisores del VIH.

Este modelo centrado en las conductas individuales de riesgo parte del supuesto de que las personas son sujetos racionales, que consideran los costos y los beneficios de las diferentes alternativas de actuación en un momento dado con la información que tienen a su alcance. Es por esto que, inicialmente, los programas se concentraron en identificar los factores individuales que influyen en la transmisión del
VIH. A las personas se les pedía que informaran sobre sus creencias, sus actitudes y prácticas individuales, y, del conjunto de respuestas, mediante análisis verticales, se sacaban conclusiones, se agrupaban y se categorizaban los denominados factores de riesgo(3).

Se pasaba luego a la atribución de dichos factores a las personas, a la definición de las características epidemiológicas que son relevantes para constituir los denominados grupos de riesgo. En lo que se refiere a sexo más seguro, el modelo considera que una persona mide el riesgo presente de una determinada relación sexual, juzga sobre la eficacia y costo de las medidas de protección y toma decisiones acordes sobre qué hacer en dicha situación como un acto voluntario para proteger la propia salud; el énfasis está puesto en procesos mentales individuales subyacentes a la toma de decisiones.

La metodología de los estudios epidemiológicos CACP (conocimientos, actitudes, creencias y prácticas) permitía configurar procesos educativos y de difusión de información que se constituyeron en la finalidad de sus estrategias. Para sus gestores e impulsores, los aspectos evidenciados en las encuestas eran indispensables para lograr cambios conductuales individuales, mediante la intervención de corte cognitivo sobre la información, la percepción del riesgo, la percepción sobre el control del propio comportamiento, la confianza en sí mismo y las actitudes de las distintas personas. El énfasis estaba en la percepción o falta de percepción de los individuos, lo cual actuaba como disparador de un cambio hacia conductas saludables(4).

Podemos afirmar que, hasta finales de 1989, las políticas de información pública y las campañas de prevención tenían dos objetivos: el primero, consolidar el componente educativo; el segundo, fortalecer las acciones en comuni- 
cación. Estos eran los criterios principales a través de los cuales se podría conseguir un cambio y lograr respuestas adecuadas de los diferentes actores sociales. Con el transcurrir del tiempo se evidenció la poca efectividad de sus estrategias, motivo por el cual se llegó a considerar que los modelos basados en factores ligados a la percepción y comportamiento del individuo eran insuficientes en la lucha mundial contra el SIDA(5).

\section{Modelo antropológico-cultural}

Ya para 1991 se puede percibir la superación de la perspectiva biologicista y biomédica fundamentada en el enfoque de riesgo. Por esta razón, las nuevas reflexiones tuvieron en cuenta los contextos ambientales y socioculturales donde se inscriben las personas. Este modelo hace hincapié en los significados que los sujetos les dan a las prácticas preventivas y de riesgo, a partir de su pertenencia a contextos culturales específicos. El cambio de paradigma se fundamentó en la insuficiencia del modelo anterior y en el examen del entorno personal y social de los individuos, lo cual permitió la inclusión de los diversos sentidos, escenarios y grupos de población en los diseños de programas. En este modelo se toman en cuenta las normas y valores sociales y se analiza su configuración específica en las diferentes culturas o subculturas.

Es decir, se consideró que la vulnerabilidad no era equiparable con una mera probabilidad (riesgo epidemiológico), sino que se encontraba determinada también por una combinación de factores culturales y sociales que influyen en la conducta de los individuos. Este cambio de perspectiva se tradujo en un replanteamiento de los factores de riesgo, que modificó la comprensión de la epidemia y la dimensionó como un problema social. En consecuencia, los programas abandonaron la categoría de grupo de riesgo por ser estrecha, prescriptiva y segre- gacionista para cambiarla por la de "comportamiento de riesgo"(6).

Con esta nueva categoría se pretende acabar con los prejuicios y la discriminación social hacia la orientación homosexual. La transmisión de la infección por VIH se convirtió en un acontecimiento que compete a todos los tipos de orientaciones y prácticas sexuales; de esta manera, se logró orientar las estrategias preventivas hacia la inclusión de las relaciones sociales y eróticas "para llegar a hombres que podrían no responder a los mensajes y programas dirigidos a la comunidad homosexual". Esto permitió caracterizar el rol que juegan las personas en el control del VIH, toda vez que para lograr el éxito de las campañas se requiere la aceptación de una definición pública de riesgo y peligro y que los individuos consientan en el control de su propio comportamiento(7,8).

El empleo de la categoría "comportamiento de riesgo" presenta, al mismo tiempo, un aspecto positivo y uno negativo. En relación con el aspecto positivo, hablar de comportamiento de riesgo significa incorporar los peligros relacionados con el VIH a la realidad social dentro del universo móvil, dinámico e incierto del erotismo. El elemento negativo está asociado a la reducción del comportamiento de riesgo a la enumeración de las prácticas y conductas que se constituyen en factores predisponentes de transmisión del VIH, ocasionando la generalización de los comportamientos al no relacionarlos con las situaciones particulares de las personas. La taxonomía de los comportamientos de riesgo no permite ofrecer una explicación de las relaciones entre éstos y los contextos donde los individuos discuten y negocian sus experiencias eróticas. Tampoco logra dar cuenta de las decisiones, riesgosas o no, presentes en las situaciones reales y, mucho menos, delinear las características del grupo social al que pertenecen los individuos y desde donde ellos interactúan. 
Reconocer el aspecto positivo y negativo de estas campañas de prevención sirvió de referente para transitar a otra fase del modelo, que exigía apreciar al riesgo no como un dato epidemiológico sino como un fenómeno social y psíquico que debía caracterizarse desde los elementos socioculturales y contextuales; ello para entender que la aparición del riesgo y las percepciones que los individuos tenían sobre éste se construyen desde y dentro de las situaciones en las cuales los sujetos valoran y desarrollan sus prácticas sexuales. En consecuencia, las nuevas propuestas de intervención, desde este modelo, consideraron los comportamientos de riesgo desde los diversos sentidos, escenarios y grupos de socialización en los cuales se inscriben e interactúan las personas, intentaron modificar las normas y los valores que potencian los riesgos e impiden los comportamientos preventivos, y alentaron la promoción de aquellas normas y valores que hacen viables los comportamientos saludables.

La transformación de las percepciones se debió, en parte, a los aportes de los trabajos e investigaciones realizados desde las Ciencias Sociales, que se constituyeron en los elementos claves para caracterizar la complejidad de las conductas sexuales y exponer que las normas racionales para el control de los riesgos eran infructuosas. Su gran contribución fue integrar una mirada construccionista del diseño de los programas de prevención, permitiendo particularizar las estrategias y, asimismo, adaptar las intervenciones de acuerdo con las singularidades, las particularidades y el entorno social de los individuos

Sin embargo, al igual que en el modelo anterior, se pueden señalar algunas críticas. Dos de ellas se refieren a la tendencia culturalista y/o psicologista en las explicaciones de la pandemia. Esto hizo necesario avanzar hacia una concepción que involucrara los aspectos estructurales como elementos clave para com- prender y trabajar frente a la infección por el VIH(9-11).

\section{Modelo político-económico}

Al final de la década de los noventa y al inicio del siglo XXI surge el modelo político-económico. Éste parte de la idea de que las desigualdades económicas y sociales están en la base de la epidemia y sostiene que ellas constituyen importantes obstáculos estructurales para los cambios de comportamiento hacia conductas saludables. Este modelo desplaza la idea de riesgo individual por el concepto de vulnerabilidad social e individual y exclusión social. Las intervenciones preventivas están dirigidas hacia el activismo de base comunitaria, movilizado para la defensa de los derechos de las minorías y de las personas afectadas. Se deja de analizar el riesgo percibido en términos de comportamiento individual para centrarse en la vulnerabilidad estructurada y condicionada socialmente.

Este modelo nace de la evidencia de que los sujetos se conducen no sólo a partir de sus actitudes individuales y de las pautas sociales, sino también en función de los recursos estructurales capitales y simbólicos con los que cuentan. Como lo señala Pierre Bourdieu, estos recursos se encuentran distribuidos de manera desigual en las personas y están atravesados profundamente por diferencias de clase(12).

Las intervenciones estructurales, la movilización comunitaria y el empoderamiento colectivo derivados del modelo tienen en común su enfoque crítico sobre las causas económicas, políticas, culturales y sociales que determinan la dinámica de la epidemia por el VIH/ SIDA. El riesgo individual se encuentra inscrito en una estructura que hace que algunos grupos sean más vulnerables que otros. Por lo tanto, las acciones propuestas desde este modelo son necesariamente de largo alcance y más amplias que aquellas que sólo buscan cambios 
de comportamiento individuales: están dirigidas incluso a cambiar políticas que favorezcan la desigualdad y la injusticia.

El modelo político-económico de prevención, al desplazar el riesgo individual hacia el de vulnerabilidad social, lleva a una reacción frente a la epidemia del VIH/SIDA, incentivando un cambio social capaz de hacer posible la reforma de las estructuras de desigualdad. Según esta perspectiva, la reacción social debe apuntar a transformar las relaciones de género que hacen más vulnerables a las mujeres, a reformar una estructura económica que liga al SIDA cada vez más con la pobreza y los grupos marginales, a revertir la exclusión de la que son víctimas los usuarios de drogas y a superar la estigmatización de la homosexualidad que hace que los HSH continúen siendo víctimas preferenciales de la epidemia. El modelo propone, además, que la prevención debe integrarse a la atención de las personas, no sólo por razones humanitarias sino también de salud pública, lo cual trae como consecuencia la propuesta de integralidad en los programas de prevención-atención.

Los riesgos de este modelo pueden situarse en el determinismo económico y social dado a la problemática del VIH/SIDA y el poco margen de maniobra que puede otorgar a los individuos, quienes, a pesar del peso de las categorías estructurales, siempre tienen la posibilidad de ejercer ciertos niveles de resistencia frente a éstas.

Los elementos propuestos en los dos primeros modelos se han incorporado, en mayor o menor medida, en la formulación de las campañas realizadas internacionalmente en los últimos años, como lo ilustran las campañas de prevención lideradas por ONUSIDA para 2000-2001 ("Los hombres marcan la diferencia") y 20022003 ("Viva y deje vivir"), que señalaron, en la primera de ellas, que los hombres - tanto homo, hetero como bisexuales- son determinantes en la transmisión del VIH y, en la segunda, que se debe superar la estigmatización y el rechazo a las personas viviendo con VIH/SIDA para lograr un control adecuado de la epidemia(13).

En particular la campaña "Los hombres marcan la diferencia" consideró que las relaciones sexuales entre hombres son una realidad presente en nuestra sociedad y que las prácticas homosexuales se pueden experimentar entre dos homosexuales, entre un homo y un heterosexual, entre un bisexual y un homosexual y entre dos heterosexuales. Igualmente, que estas relaciones se pueden dar o no con el reconocimiento de una identidad homoerótica por parte de los participantes. Se sugiere, entonces, hablar de una homosexualidad permanente y de una circunstancial, y se prefiere utilizar el término hombres que tienen sexo con hombres (HSH). Al respecto, es pertinente señalar que para que esta categoría sea útil y válida, dentro de un programa de prevención, deberá estar sustentada desde una perspectiva construccionista del género y la sexualidad, y llenarse de contenidos socioculturales alejados de los discursos esencialistas. Para que las acciones y políticas públicas puedan hacerse cargo de estas nociones de sexualidad, deben dar cuenta de la naturaleza compleja de su objeto (ligado a prácticas subjetivas sociales), que escapa a las definiciones clásicas de la epidemiología y la salud pública. Está en construcción la incorporación de los elementos del tercer modelo a las acciones encaminadas a disminuir la epidemia(14).

\section{Lecciones aprendidas}

Con base en los estudios revisados, podemos concluir que la relación entre comportamiento sexual e identidad de género es de una gran complejidad y que las formas a través de las cuales se construyen las identidades sexuales en distintos contextos dependen en gran 
parte de las categorías y clasificaciones disponibles en cada sociedad y cultura para abordar la sexualidad. El foco de los estudios se ha desplazado del comportamiento sexual en sí mismo hacia las situaciones sociales en las cuales se produce dicho comportamiento y hacia las normas culturales que lo organizan. Se ha hecho evidente que categorías como homo o heterosexualidad no reflejan la diversidad y complejidad de las experiencias sexuales vividas, $\mathrm{y}$ se ha disociado el comportamiento homo o heterosexual de un sentido diferenciado de identidad de género(15).

En lo concerniente a las acciones educativas implementadas en América Latina, diversas investigaciones producidas en los últimos años han evidenciado problemas de diferente índole, entre los cuales podemos identificar los siguientes:

- Los mensajes de prevención pierden de vista la complejidad de las motivaciones de los hombres y mujeres para tener relaciones sexuales, así como los sentidos emocionales que se asignan a éstas.

- Los planteamientos del sexo protegido o del uso del condón pueden resultar ofensivos por los supuestos que connota (de infidelidad, de tener infecciones de transmisión sexual, de desconfianza en la pareja) o por la complejidad de las interpretaciones que genera.

- El sexo no protegido se ve como facilitador de una mayor cercanía e intimidad con la pareja "estable" y se asumiría como una práctica normal, libre de riesgo.

- El cálculo del riesgo de lo(a)s compañero(a)s sexuales se sigue haciendo a partir de su apariencia de persona "sana" o "enferma", y esto induciría a tener sexo sin protección con muchas de estas personas que lucen aparentemente sanas.
- La baja autoestima, así como la clandestinidad de las experiencias sexuales (facilitadas por alcohol o drogas) a las que se enfrentan muchos hombres y mujeres, afectan la capacidad cognitiva y emocional de los sujetos para adoptar conductas preventivas.

- La dificultad de la incorporación del uso del condón en muchos de los escenarios de la sexualidad, en tanto en dichos escenarios otros riesgos estarían presentes y serían valorados como más importantes (como la integridad física, el riesgo de ser detenidos y encarcelados, el riesgo de perder la oportunidad del encuentro sexual).

- No todas las personas están listas para mensajes directos y contundentes y, por tanto, muchas requieren mensajes o aproximaciones elípticas.

En la actualidad se ha tomado conciencia de que la infección se encuentra en una fase temprana de desarrollo y sigue sin estar claro cómo evolucionará en el largo plazo. Se han identificado y probado muchos enfoques eficaces para la prevención del VIH y se ha reconocido la necesidad de hacer particular hincapié en las poblaciones en situación de vulnerabilidad, en particular los HSH. Sin embargo, dado que la distribución del riesgo y la vulnerabilidad varía mucho de una sociedad a otra, igual que la capacidad de localizar y trabajar con la población vulnerable, no hay un enfoque único de prevención que resulte eficaz en todas partes y con todas las personas. Para producir y mantener cambios de comportamiento eficaces a gran escala es menester que los programas específicos de prevención abarquen muchos elementos, y sean elaborados a partir de las propuestas de cada una de las poblaciones destinatarias, para tratar sus necesidades específicas y los factores que influyen sobre los cambios de comportamiento. 
Una estrategia de intervención en VIH/SIDA debería estar constituida por un discurso que promueva el respeto y la equidad de género, revalorice la sexualidad y permita la libre expresión y ejercicio de las diversas orientaciones. Igualmente, se pretende cuestionar aquellos factores que reproducen actitudes y conductas negativas e intolerantes hacia las identidades homosexuales y se apunta a la desestigmatización de esta orientación(16).

La inversión en la prevención del VIH evita un incalculable sufrimiento humano y también sus consecuencias sociales y de desarrollo, independientemente de las tasas de prevalencia de cada país. El aumento de la incidencia del VIH sólo podrá detenerse con una expansión masiva de los esfuerzos de promoción y prevención. Es en este sentido que los programas de prevención se tornan fundamentales, y deben continuarse con cada generación de hombres y mujeres que alcanzan la madurez sexual y necesitan aprender a vivir satisfactoriamente con el panorama de la infección VIH/SIDA en sus vidas(17).

\section{Referencias}

1. UNAIDS. Report on the global HIV/AIDS epidemic 2003.

2. Garcia JM, Walker N, Cuchi P, et al. Status of the HIV/AIDS epidemic and methods to monitor it in the Latin America and Caribbean region. AIDS 2002; 16 (suppl. 3): S3-S12.

3. Sevilla E. Modelos analíticos de las ciencias sociales para prevención de infección por VIH En: Cinco estudios antropológicos sobre el mal de amores en la ciudad de Cali. Documento de trabajo No 44. Cali: Cidse - Universidad del Valle; 1998.

4. Calvez M. Los usos sociales del riesgo. Elementos de análisis cultural del SIDA. San Martín: Editorial Universitaria; 1998.

5. Sevilla E. Dueños de sí y de sus deseos: Estudio sobre la Sexualidad de los Colombianos y su vulnerabilidad al VIH. Manuscrito, informe de Investigación. Cali: Cidse - Universidad del Valle; 1995.

6. Sevilla E. Perfiles de la sexualidad a propósito de las diferencias entre hombres y mujeres en Colombia. Estudios Demográficos y Urbanos 1997; 12: 1-2.

7. Lamas M. Explicar la homofobia. Letra S 1996; 2.

8. Kimmel M. Homofobia, temor, vergüenza y silencio en la identidad masculina. En: Valdés T, Olavarría J, (eds.) Masculinidades. Poder y crisis. Santiago de Chile: Isis Internacional - Flacso; 1997.

9. Finkielkraut A, Bruckner P. El nuevo desorden amoroso. Barcelona: Anagrama; 1979.

10. Fuller N. No uno si no muchos rostros. Identidad masculina del Perú urbano. En: Viveros M, Olavarría $\mathrm{J}$, Fuller N. Hombres e identidades de género. Investigaciones desde América Latina. Bogotá: Ed. CES - Universidad Nacional; 2001.

11. Viveros M. Género y salud reproductiva en Colombia. En: Franco S, (ed.) La Salud Pública hoy: Enfoques y dilemas contemporáneos en Salud Pública. Bogotá: Universidad Nacional de Colombia; 2003: 481-93. 
Modelos de prevención en la lucha contra el VIH/SIDA - J. Estrada M.

12. Téllez G. Pierre Bourdieu. Conceptos básicos y construcción socioeducativa. Claves para su lectura. Bogotá: Universidad Pedagógica Nacional; 2002.

13. ONUSIDA. El SIDA y las relaciones sexuales entre varones. Mayo de 2000.

14. Giddens A. La transformación de la intimidad. Sexualidad, amor y erotismo en las sociedades modernas. Madrid: Ediciones Cátedra; 2000.

15. Scott J. El género como categoría útil para un análisis histórico. En: Lamas M, (comp.) La construcción de la diferencia sexual. México: Editorial PUEG/UNAM; 2001.

16. ONUSIDA. Resumen mundial de la epidemia de VIH/SIDA. Diciembre de 2003.

17. Castro A. Determinantes socio-políticos de la infección por VIH: violencia estructural y culpabilización de la víctima. Conferencia presentada en el $2^{\circ}$ Foro Latinoamericano de VIH/SIDA. La Habana: abril 10 de 2003.

Recibido: 13 de enero de 2006

Aceptado: 01 de febrero de 2006 